\title{
Cellulose acetate/P4VP-b-PEO membranes for the adsorption of electron deficient pharmaceutical compounds
}

Laura Penabad-Peña ${ }^{1,2}$, Jairo Herrera-Morales ${ }^{1,2}$, Miguel Betancourt ${ }^{1,2}$, Eduardo Nicolau ${ }^{1,2}$

${ }^{1}$ Department of Chemistry, University of Puerto Rico, Río Piedras Campus, P.O. 23346, San Juan, Puerto Rico 00931-3346, United States

${ }^{2}$ Molecular Sciences Research Center, University of Puerto Rico, 1390 Ponce De Leon Avenue, Suite 2, San Juan, Puerto Rico 00931-3346, United States

Table of contents

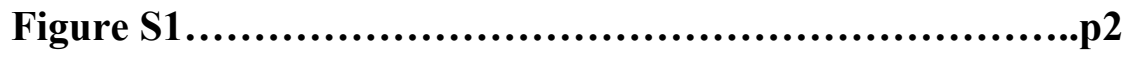

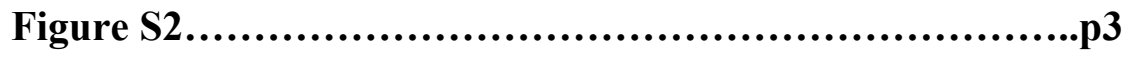

Figure S3..................................................p3

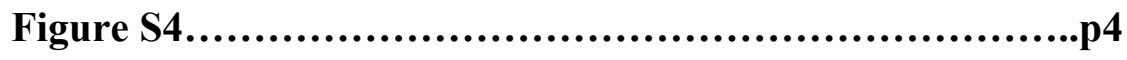

Figure S5...................................................p5

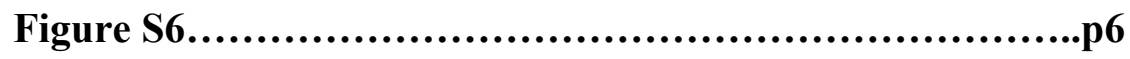


<smiles>COc1ccc2[nH]c(S(=O)Cc3ncc(C)c(OC)c3C)nc2c1</smiles><smiles></smiles><smiles>COc1ccc2[nH]c(-c3nc(CSO)c(C)c(OC)c3C)nc2c1</smiles><smiles>COc1ccc2nc3n(c2c1)SCc1ncc(C)c(OC)c1C3</smiles><smiles></smiles>

a

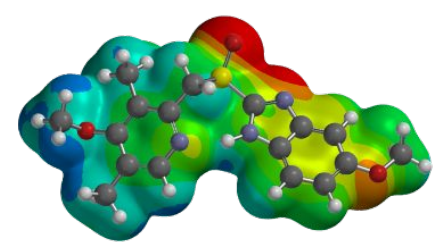

b

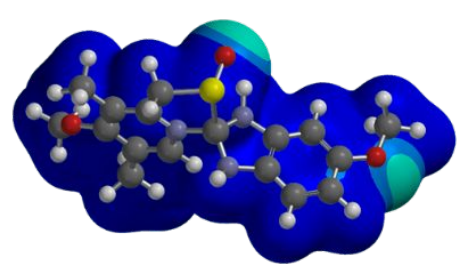

C

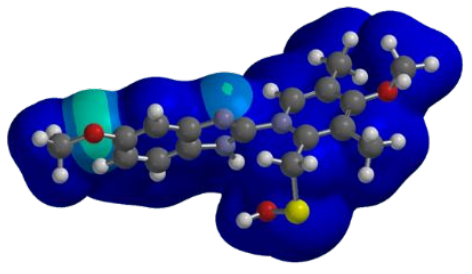

d

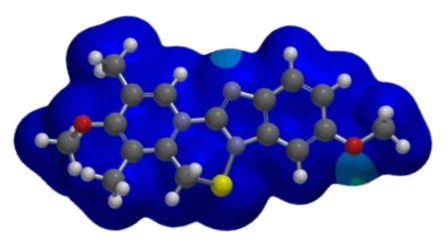

e

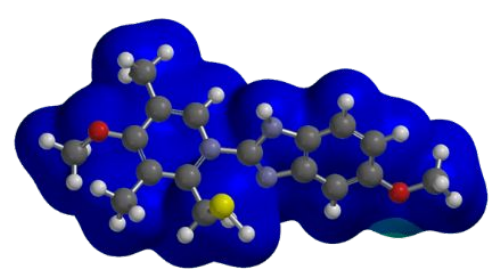

Figure S1: Omeprazole (OMZ) molecules and intermediary species electron density maps. The figure shows (a) pristine OMZ with its ED map (b-e) possible OMZ degradation intermediary species OMZ ID with their respective ED maps.

The figure shows that both the electron density of the OMZ molecule and, specifically, its intermediates have highly deactivated rings as well as the spatial configuration that would allow for the $\pi-\pi$ interactions with the pyridine ring from the P4VP-PEO. 

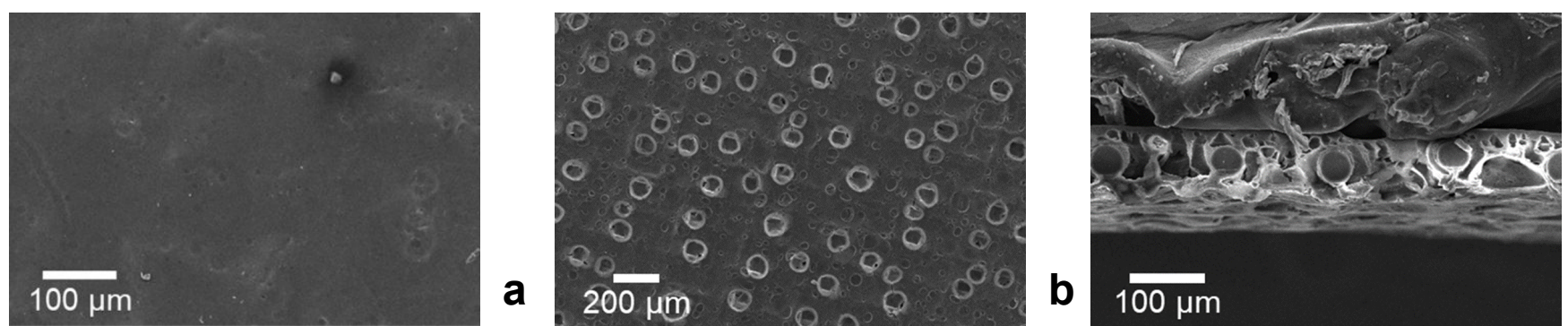

Figure S2: SEM photos for 3\% P4VP-PEO. The figure shows the (a) top surface, (b) bottom surface, (c) cross-section.

This characterization is consistent with the one described in the manuscript. The presence of P4VPPEO is related to the formation of superficial and hierarchical pores.
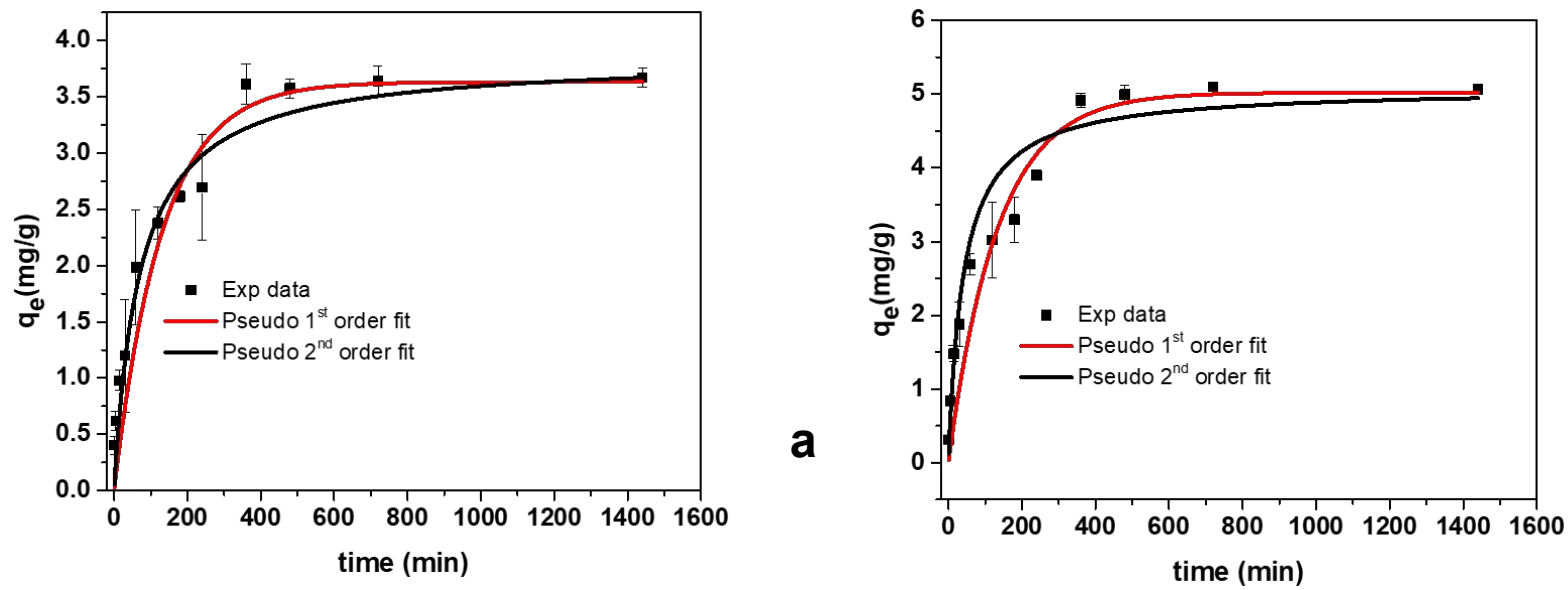

b

Figure S3: Kinetic experiments for composite membranes with varying P4VP-PEO concentrations. The figure shows (a) 1\%P4VP-PEO and (b) 3\% P4VP-PEO.

This experiment was performed in $30 \mathrm{ppm}$ of SMX for 24 hours. The data indicates that tripling the amount of P4VP-PEO in the membrane only gives way to a $\sim 40 \%$ adsorption capacity increase. Thus, the remainder of the experiments were carried out with 1\% P4VP-PEO. 

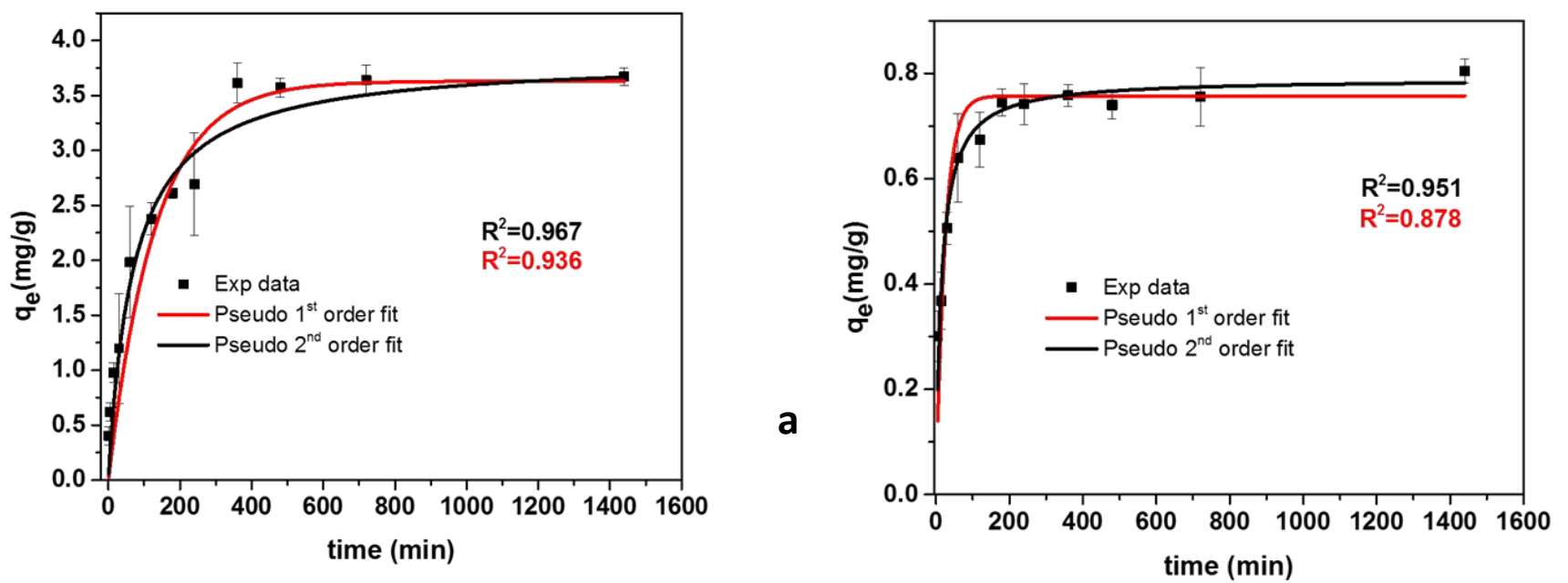

b
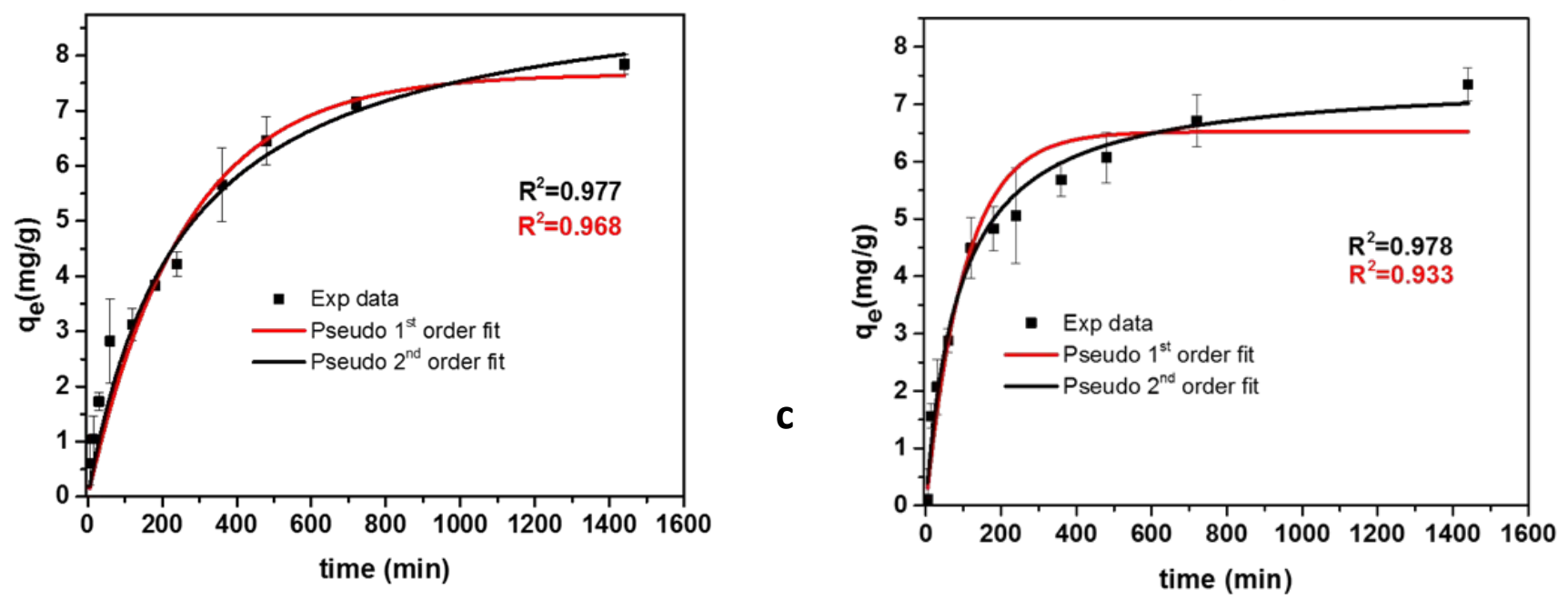

d

Figure S4: Kinetic experiments for the composite membrane: Adsorption (qe) as a function of time (min).
(a) SMX
(b) SDZ, (c)
(c) OMZ covered from light,

\section{(d) OMZ exposed to light}

The contact time experiments were a 24 hour- batch assay performed in $30 \mathrm{ppm}$ of the pharmaceuticals under study. All of the graphs in the figure display a higher $\mathrm{R}^{2}$ value for pseudo $2^{\text {nd }}$ order adsorption (chemisorption) which is consistent with the EDA/ $\pi-\pi$ interactions taking place between the contaminants and the P4VP-PEO. It should be noted that both OMZ species degraded into intermediary species at different rates while the experiment was taking place. 

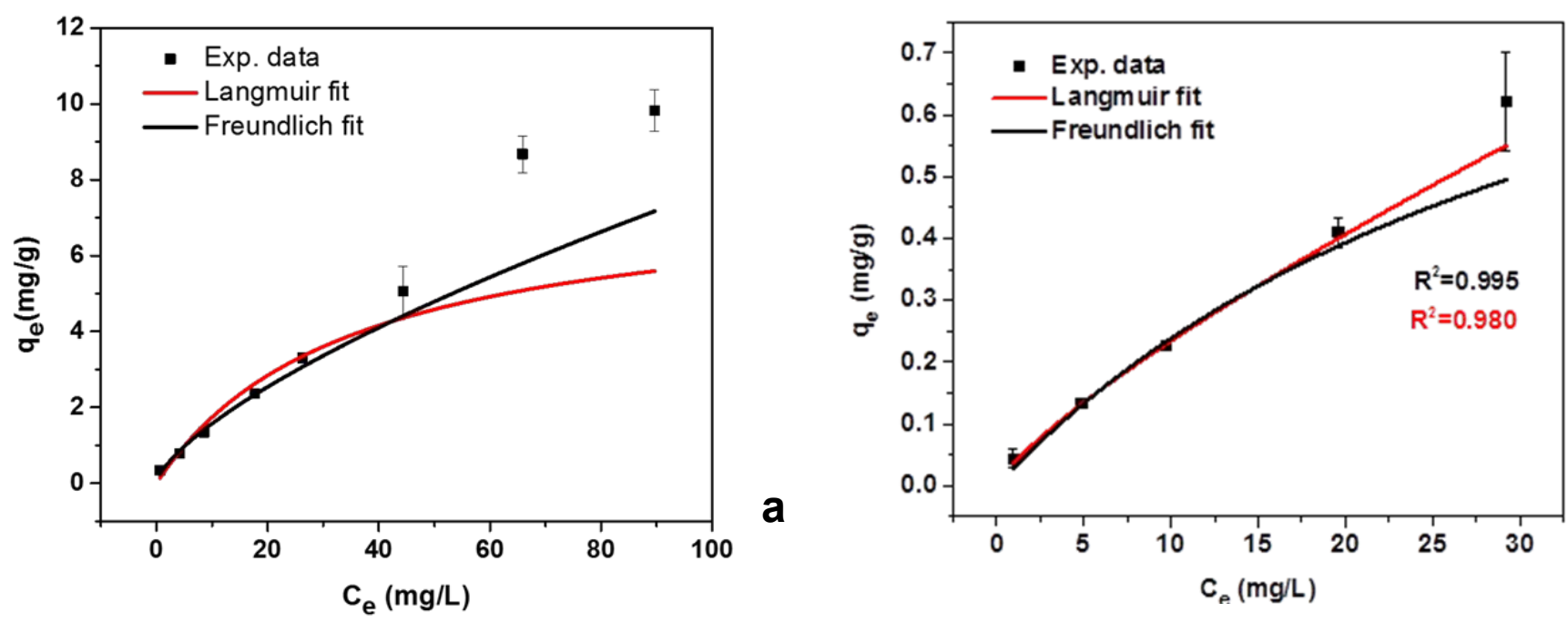

b
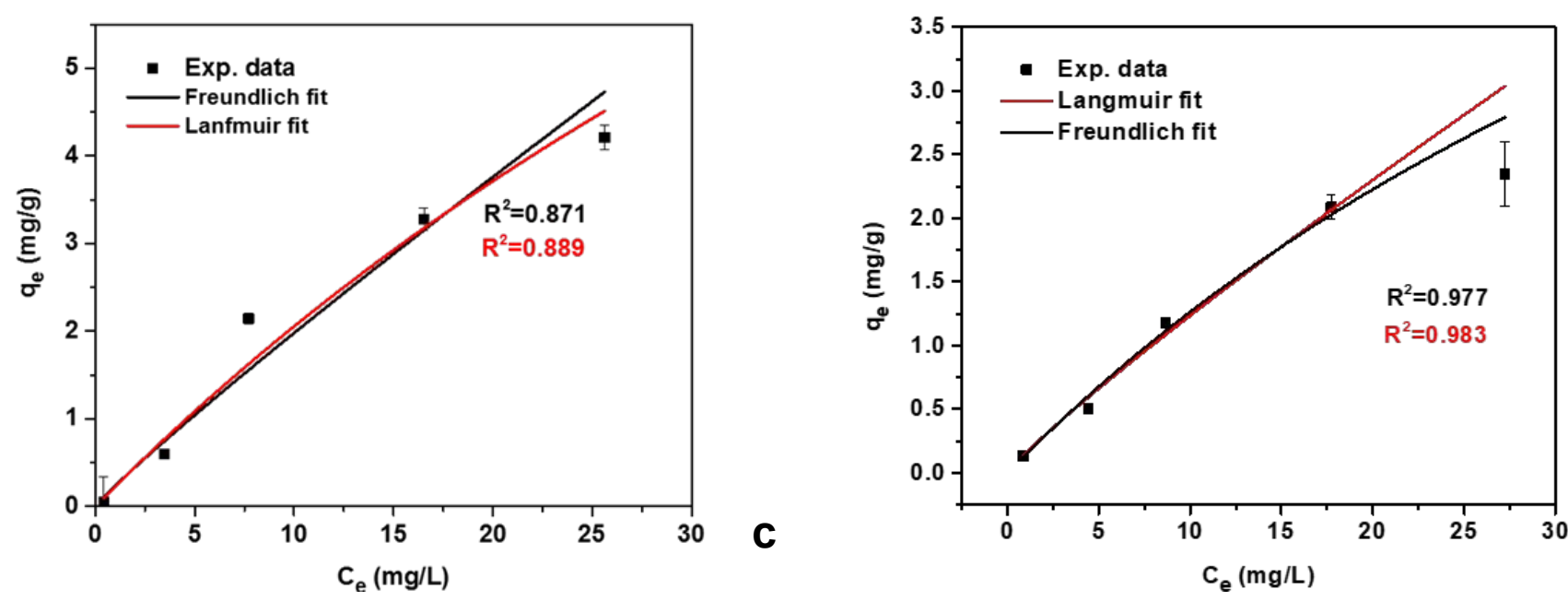

Figure S5: Isotherm experiments for the composite membrane: Adsorption ( $q e)$ a a function of equilibrium concentration $\left(C_{e}\right)$. (a) $S M X$, (b) SDZ, (c) OMZ covered from light, (d) OMZ exposed to light

The isotherm experiments were a 4-hour batch assay. All of the graphs are fitted with Langmuir and Freundlich fits in an attempt to determine the distribution of adsorbing sites on the surface of the composite membrane. The $\mathrm{R}^{2}$ value of the fits for SMX is too low to be statistically significant. However, the data might suggest a breakthrough adsorbing behavior at concentrations above $30 \mathrm{ppm}$. The other graphs display very similar $\mathrm{R}^{2}$ values in each set of data, and there is no apparent conclusive trend in either of the fits 


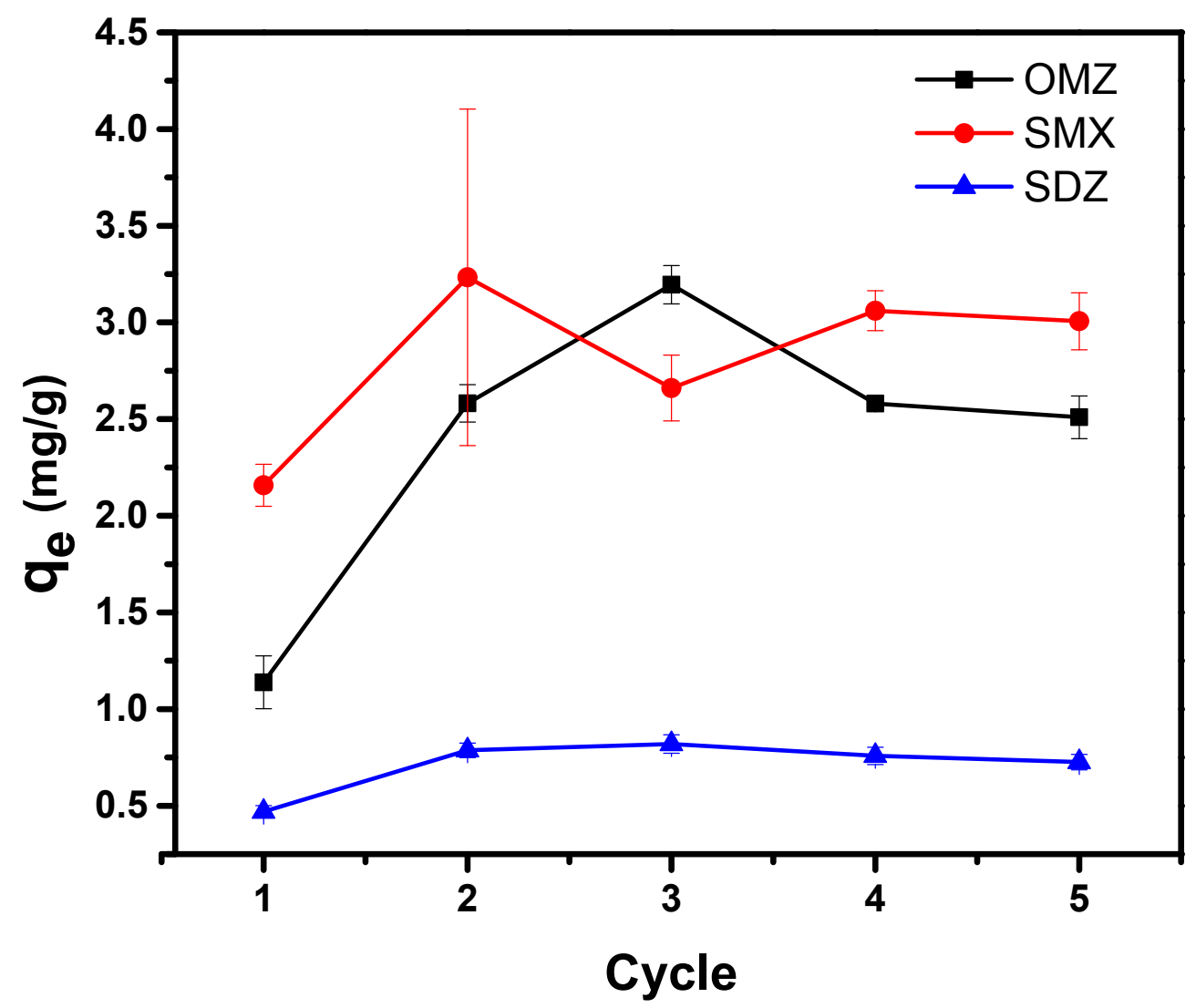

Figure S6: Reusability cycles for the composite membrane for each pharmaceutical.

The reusability cycles show a prominent increase of adsorption capacity from the first cycle to the second cycle, and then a consistency is maintained through the next 2-5 cycles. 